Appl. Set-Valued Anal. Optim. 2 (2020), No. 2, pp. 235-253

Available online at http://asvao.biemdas.com

https://doi.org/10.23952/asvao.2.2020.2.08

\title{
BREGMAN COMMON SKEW-ATTRACTIVE POINT THEOREMS FOR SEMIGROUPS OF NONLINEAR MAPPINGS IN BANACH SPACES
}

\author{
LALEH ESLAMIZADEH, ESKANDAR NARAGHIRAD*
}

Department of Mathematics, Yasouj University, Yasouj 75918, Iran

\begin{abstract}
In this paper, we prove Bregman common attractive point and Bregman common skew-attractive point theorems for a semitopological semigroup $S$ of Bregman type nonexpansive (not necessarily continuous) mappings acting on a closed convex subset $C$ of a Banach space $E$. Our results propose the existence of Bregman common attractive points and Bregman common skew-attractive points for nonlinear mappings in a Banach space $E$.
\end{abstract}

Keywords. Bregman function; Fixed point; Bregman skew-attractive point; Semigroup; Invariant mean.

\section{INTRODUCTION}

Let $S$ be a semigroup. We denote by $l^{\infty}(S)$ the Banach space of all bounded real-valued functions on $S$ with supremum norm. For each $s \in S$, we define the left and right translation operators $l(s)$ and $r(s)$ on $l^{\infty}(S)$ by

$$
(l(s) f)(t)=f(s t) \quad \text { and } \quad(r(s) f)(t)=f(t s)
$$

for each $s \in S$ and $f \in l^{\infty}(S)$, respectively. Let $X$ be a subspace of $l^{\infty}(S)$ containing 1 . An element $\mu$ in the dual space $X^{*}$ of $X$ is said to be a mean on $X$ if $\|\mu\|=\mu(1)=1$. For $s \in S$, we can define a point evaluation $\delta_{s}$ by $\delta_{s}(f)=f(s)$ for each $f \in X$. If $X$ is a translation invariant subspace of $l^{\infty}(S)$ (i.e., $l(s) X \subset X$ and $r(s) X \subset X$ for each $s \in S$ ) containing 1 . Then a mean $\mu$ on $X$ is said to be left invariant (res. right invariant) if

$$
\mu(l(s) f)=\mu(f)(\text { res. } \mu(r(s) f)=\mu(f))
$$

for each $s \in S$ and $f \in X$. A mean $\mu$ on $X$ is said to be invariant if $\mu$ is both left and right invariant. $S$ is said to be left (res. right) amenable if $X$ has a left (res. right) invariant mean. $S$ is amenable if $S$ is left and right amenable. In this case, $l^{\infty}(S)$ also has an invariant mean. A net $\left\{\mu_{\alpha}\right\}_{\alpha \in I}$ of means on $X$ is said to be asymptotically left (resp. right) invariant if

$$
\lim _{\alpha}\left(\mu_{\alpha}(l(s) f)-\mu_{\alpha}(f)\right)=0\left(\operatorname{resp} . \lim _{\alpha}\left(\mu_{\alpha}(r(s) f)-\mu_{\alpha}(f)\right)=0\right)
$$

for each $f \in X$ and $s \in S$, and it is said to be left (resp. right) strong asymptotically invariant (or strong regular) if

$$
\lim _{\alpha}\left(\mu_{\alpha}\left\|l^{*}(s) \mu_{\alpha}-\mu_{\alpha}\right\|=0\left(\text { resp. } \lim _{\alpha}\left(\left\|r^{*}(s) \mu_{\alpha}-\mu_{\alpha}\right\|=0\right)\right.\right.
$$

${ }^{*}$ Corresponding author.

E-mail addresses: lale.eslami1393@gmail.com (L. Eslamizadeh), eskandarrad@gmail.com, esnaraghirad@yu. ac.ir (E. Naraghirad).

Received June 25, 2020; Accepted August 1, 2020.

(C)2020 Applied Set-Valued Analysis and Optimization 
for each $s \in S$, where $l^{*}(s)$ and $r^{*}(s)$ are the adjoint operators of $l(s)$ and $r(s)$, respectively. Such nets were first introduced by Day in [7] and called weak* invariant and norm invariant, respectively.

A semigroup $S$ with an identity $e$ is called left reversible if any two closed right ideals of $S$ have non-void intersection, i.e., $\bar{a} S \cap \overline{b S} \neq \emptyset$ for $a, b \in S$.

Let $S$ be a semigroup and $C$ be a closed and convex subset of $E$. A family $\mathcal{F}:=\{T(s): s \in S\}$ is called a representation of $S$ as Bregman relatively nonexpansive mappings on $C$ if $T(s)$ is Bregman relatively nonexpansive and $T(s t)=T(s) T(t)$ for each $s, t \in S$. We denote by $F(\mathcal{F})$ the set of common fixed points of $\mathcal{F}$, i.e.,

$$
F(\mathcal{F})=\cap_{s \in S} F(T(s))=\cap_{s \in S}\{x \in C: T(s) x=x\} .
$$

We assume that $X$ is a subspace of $l^{\infty}(S)$ containing 1 and the function $s \mapsto\left\langle T(s) x, x^{*}\right\rangle$ is contained in $X$ for each $x \in C$ and $x^{*} \in E^{*}$. Then there exists a unique point $x_{0}$ of $E$ such that $\mu\left\langle T() x,. x^{*}\right\rangle=\left\langle x_{0}, x^{*}\right\rangle$ for a mean $\mu$ on $X, x \in C$ and $x^{*} \in E^{*}$. We denote such a point $x_{0}$ by $T_{\mu} x$. Note that $T_{\mu} x$ is contained in the closure of the convex hull of $\{T(s) x: s \in S\}$ for each $x \in C$, (see, for more details, [10]).

Given a semitopological semigroup $S$, let $\ell^{\infty}(S)$ be the $C^{*}$-algebra of bounded complexvalued functions on $S$ with the supremum norm and pointwise multiplication. For each $s \in S$ and $f \in \ell^{\infty}(S)$, denote by $\ell_{s} f$ and $r_{s} f$ the left and right translates of $f$ by s respectively, that is, $\left(\ell_{s} f\right)(t)=f(s t)$ and $\left(r_{s} f\right)(t)=f(t s)(t \in S)$. Let $X$ be a closed subspace of $l^{\infty}(S)$ containing the constant functions and being invariant under left translations. Then a linear functional $m \in X^{*}$ is called a mean if $\|m\|=m(1)=1 ; m$ is called a left invariant mean, denoted by LIM, if $m\left(\ell_{s} f\right)=m(f)$ for all $s \in S, f \in X^{*}$. Let $C_{b}(S)$ be the space of all bounded continuous complex-valued functions on $S$. Then $C_{b}(S)$ certainly is a closed subalgebra of $l^{\infty}(S)$ containing the constant functions and being invariant under translations. Let $L U C(S)$ be the space of left uniformly continuous functions on $S$, that is, all $f \in C_{b}(S)$ such that the mappings $s \mapsto \ell_{s}(f)$ from $S$ into $C_{b}(S)$ are continuous. Then $L U C(S)$ is a $C^{*}$-subalgebra of $C_{b}(S)$ invariant under translations and contains the constant functions. When $\mathrm{S}$ is a topological group, then $L U C(S)$ is precisely the space of bounded right uniformly continuous functions on $\mathrm{S}$ as defined in [9]. The semigroup $\mathrm{S}$ is called left amenable (respectively extremely left amenable) if $L U C(S)$ has a LIM (respectively a multiplicative LIM). The theory concerning amenability of semigroups can be found in monographs [28] and [29], see also, [13, 14, 15]. A net $\left\{\mu_{\alpha}\right\}_{\alpha \in I}$ of means on $X$ is said to be asymptotically invariant if, for each $f \in X$ and $s \in S$,

$$
\mu_{\alpha}(f)-\mu_{\alpha}\left(l_{s} f\right) \rightarrow 0 \quad \text { and } \mu_{\alpha}(f)-\mu_{\alpha}\left(r_{s} f\right) \rightarrow 0 .
$$

Let $A P(S)$ be the space of all $f \in C_{b}(S)$ such that $\mathcal{L} \mathcal{O}(f)=\left\{\ell_{s} f: s \in S\right\}$ is relatively compact in the norm topology of $C_{b}(S)$, and let $W A P(S)$ be the space of all $f \in C_{b}(S)$ such that $\mathcal{L O}(f)$ is relatively compact in the weak topology of $C_{b}(S)$. Functions in $A P(S)$ (respectively $W A P(S)$ ) are called almost periodic (respectively weakly almost periodic) functions. $A P(S)$ and $W A P(S)$ are closed $C^{*}$-subalgebras of $C_{b}(S)$ invariant under translations and contains the constant functions.

Suppose that $C$ is a subset of a Banach space $E$ and that $\mathcal{S}=\left\{T_{s}: s \in S\right\}$ is a representation of $S$ on $C$. Let $x \in C$ be such that $\left\{T_{s} x: s \in S\right\}$ is bounded. Then each $f \in E^{*}$ defines an element $f_{x} \in \ell^{\infty}(S)$ for which $f_{x}(s)=\left\langle T_{s} x, f\right\rangle$ for $s \in S$. If $s \mapsto T_{s} x: S \rightarrow C$ is continuous when $C$ is equipped with the weak topology of $E$, then $f_{x} \in C_{b}(S)$; if the action of $S$ on $C$ is weakly jointly 
continuous and $\left\{T_{s} x: s \in S\right\}$ is weakly relatively compact, then $f_{x} \in L U C(S)$. If the action of $S$ on $C$ is weakly separately continuous and weakly equicontinuous continuous and $\left\{T_{S} x: s \in S\right\}$ is weakly relatively compact, then $f_{x} \in A P(S)$ [13](Lemma 3.1). Finally, if the action of $S$ on $C$ is weakly separately continuous and weakly quasi-equicontinuous and $\left\{T_{s} x: s \in S\right\}$ is weakly precompact, then $f_{x} \in W A P(S)$ [19] (Lemma 3.2). Here we recall that a representation $\mathcal{R}=\left\{T_{S}: s \in S\right\}$ on a Hausdorff space $X$ is quasi-equicontinuous if $\overline{\mathcal{R}} p$, the closure of $\mathrm{S}$ in the product space $X^{X}$, consists of only continuous mappings. In other words, the representation is quasi-equicontinuous if, for any net $\left\{s_{i}\right\}_{i \in I} \subset S$, whenever $T_{s_{i}}(z) \rightarrow T(z)$ for each $z \in X, T$ is a continuous mapping from $X$ into $X$. Now let $X$ be a closed subspace of $\ell^{\infty}(S)$ containing the constant functions. Let $\mathcal{R}=\left\{T_{S}: s \in S\right\}$ be a representation of $S$ on $C$ as above. Suppose that $x \in C$ such that $\left\{T_{s} x: s \in S\right\}$ is bounded, and suppose that $f_{x} \in X$ for each $f \in E^{*}$. For any mean $\mu \in X^{*}$, we can define $T_{\mu} \in E^{* *}$ by

$$
\left\langle T_{\mu} x, f\right\rangle=\mu\left(f_{x}\right)
$$

$T_{\mu} x$ is clearly well-defined. If $\left\{T_{s} x: s \in S\right\}$ is precompact, then $T_{\mu} x$ is weak* continuous. So $T_{\mu} x \in E$ in this case. For details concerning the geometry of Banch spaces, we refer the reader to [37].

1.1. Some facts about gradients. For any convex function $g: E \rightarrow(-\infty,+\infty]$, we denote the domain of $g$ by $\operatorname{dom}(g)=\{x \in E: g(x)<\infty\}$. For any $x \in$ int (dom) $g$ and any $y \in E$, we denote by $g^{o}(x, y)$ the right-hand derivative of $g$ at $x$ in the direction $y$, that is,

$$
g^{o}(x, y)=\lim _{t \downarrow 0} \frac{g(x+t y)-g(x)}{t} .
$$

The function $g$ is said to be Gâteaux differentiable at $x$ if $\lim _{t \rightarrow 0} \frac{g(x+t y)-g(x)}{t}$ exists for any $y$. In this case, $g^{o}(x, y)$ coincides with $\nabla g(x)$, the value of the gradient $\nabla g$ of $g$ at $x$. The function $g$ is said to be Gâteaux differentiable if it is Gâteaux differentiable everywhere. The function $g$ is said to be Fréchet differentiable at $x$ if this limit is attained uniformly in $\|y\|=1$. The function $g$ is said to be Fréchet differentiable if it is Fréchet differentiable everywhere. It is well known that if a continuous convex function $g: E \rightarrow(-\infty,+\infty]$ is Gâteaux differentiable, then $\nabla g$ is norm-to-weak* continuous (see, for example, [4]). Also, it is known that if $g$ is Fréchet differentiable, then $\nabla g$ is norm-to-norm continuous (see [12]). The function $g$ is said to be strongly coercive if

$$
\lim _{\left\|x_{n}\right\| \rightarrow \infty} \frac{g\left(x_{n}\right)}{\left\|x_{n}\right\|}=\infty
$$

It is also said to be bounded on bounded subsets of $E$ if $g(U)$ is bounded for each bounded subset $U$ of $E$. Finally, $g$ is said to be uniformly Fréchet differentiable on a subset $X$ of $E$ if the limit (1.1) is attained uniformly for all $x \in X$ and $\|y\|=1$.

1.2. Some facts on the Bregman distance. Let $E$ be a Banach space. A function $g: E \rightarrow$ $(-\infty,+\infty]$ is said to be proper if the interior of its domain

$$
\operatorname{dom}(g)=\{x \in E: g(x)<+\infty\}
$$

is nonempty. Let $g: E \rightarrow(-\infty,+\infty]$ be a convex and Gâteaux differentiable function. Then the Bregman distance $[3,5]$ corresponding to $g$ is the function $D_{g}: \operatorname{dom}(g) \times$ int $\operatorname{dom}(g) \rightarrow \mathbb{R}$ 
defined by

$$
D_{g}(x, y)=g(x)-g(y)-\langle x-y, \nabla g(y)\rangle, \forall x, y \in E .
$$

It is clear that $D_{g}(x, y) \geq 0$ for all $x, y \in E$. When $E$ is a smooth Banach space, setting $g(x)=$ $\|x\|^{2}$ for all $x$ in $E$, we have that $\nabla g(x)=2 J x$ for all $x$ in $E$, where $J$ is the normalized duality mapping from $E$ into $E^{*}$. Hence, $D_{g}(\cdot, \cdot)$ reduces to the usual map $\phi(\cdot, \cdot)$ as

$$
D_{g}(x, y)=\phi(x, y):=\|x\|^{2}-2\langle x, J y\rangle+\|y\|^{2}, \quad \forall x, y \in E .
$$

Let $E$ be a Banach space and $g: E \rightarrow(-\infty,+\infty]$ a strictly convex and Gâteaux differentiable function. Working with the Bregman distance $D_{g}$ (1.2), the following Bregman three-point identity holds for every Banach space $E[6]$ :

$$
D_{g}(x, z)=D_{g}(x, y)+D_{g}(y, z)+\langle x-y, \nabla g(y)-\nabla g(z)\rangle, \quad \forall x, y, z \in E .
$$

In particular,

$$
D_{g}(x, y)=-D_{g}(y, x)+\langle y-x, \nabla g(y)-\nabla g(x)\rangle, \quad \forall x, y \in E \text {. }
$$

Let $E$ be a Banach space and let $C$ be a nonempty and convex subset of $E$. Let $g: E \rightarrow$ $(-\infty,+\infty]$ be a convex and Gâteaux differentiable function. Then, we know from [26] that, for $x \in E$ and $x_{0} \in C, D_{g}\left(x_{0}, x\right)=\min _{y \in C} D_{g}(y, x)$ if and only if

$$
\left\langle y-x_{0}, \nabla g(x)-\nabla g\left(x_{0}\right)\right\rangle \leq 0, \quad \forall y \in C .
$$

Furthermore, if $C$ is a nonempty, closed and convex subset of a reflexive Banach space $E$ and $g: E \rightarrow(-\infty,+\infty]$ is a strongly coercive Bregman function, then, for each $x \in E$, there exists a unique $x_{0} \in C$ such that

$$
D_{g}\left(x_{0}, x\right)=\min _{y \in C} D_{g}(y, x) .
$$

The Bregman projection $\operatorname{proj}_{C}^{g}$ from $E$ onto $C$ is defined by $\operatorname{proj}_{C}^{g}(x)=x_{0}$ for all $x \in E$. It is also well known that proj $_{C}^{g}$ has the following property:

$$
D_{g}\left(y, \operatorname{proj}_{C}^{g} x\right)+D_{g}\left(\operatorname{proj}_{C}^{g} x, x\right) \leq D_{g}(y, x)
$$

for all $y \in C$ and $x \in E$ (see $[4,26]$ for more details).

1.3. Some facts on Bregman nonexpansive type mappings. Let $C$ be a nonempty, closed and convex subset of a reflexive Banach space $E$. Let $g: E \rightarrow(-\infty,+\infty]$ be a proper, lower semicontinuous and convex function. A mapping $T: C \rightarrow C$ is said to be Bregman quasinonexpansive if $F(T) \neq \emptyset$ and

$$
D_{g}(p, T x) \leq D_{g}(p, x), \quad \forall x \in C, \forall p \in F(T) .
$$

We denote by $A_{C}^{g}(T)$ the set of Bregman attractive points of $T$; i.e.,

$$
A_{C}^{g}(T)=\left\{z \in E: D_{g}(z, T x) \leq D_{g}(x, z) \quad \forall x \in C\right\} .
$$

The theory of fixed points with respect to Bregman distances have been studied in the last twenty years and much intensively in the last decade. For some recent articles on the existence of fixed points for Bregman nonexpansive type mappings, we refer the readers to [2, 21, 22, 23, 24, 34]. Despite the fact that there are some important techniques and developments leading to the nonemptiness of the set of attractive points and construction of attractive points of nonexpansive mappings in Hilbert spaces, the corresponding approach for non-necessarily continuous mappings in general Banach spaces is largely unexplored. 
In this paper, we prove Bregman common attractive point and Bregman common skewattractive point theorems for a semitopological semigroup $S$ of Bregman type nonexpansive (not necessarily continuous) mappings acting on a closed convex subset $C$ of a Banach space $E$. Our results propose the existence of Bregman common attractive points and Bregman common skew-attractive points for nonlinear mappings in a Banach space $E$. It is well known that the Bregman distance does not satisfy either the symmetry property or the triangle inequality which are required for standard distances. So, the results of the paper improve and extend many recent results in the literature. So, the results of the paper unify and generalize many recent results in the literature; see, for example, [11, 16, 17, 18, 20, 30, 31, 32, 33, 38, 39].

\section{PRELIMINARIES}

In this section, we begin by recalling some preliminaries and lemmas which will be used in the sequel.

2.1. Some facts on conjugate functions. Let $E$ be a reflexive Banach space. For any proper, lower semicontinuous and convex function $g: E \rightarrow(-\infty,+\infty]$, the conjugate function $g^{*}$ of $g$ is defined by

$$
g^{*}\left(x^{*}\right)=\sup _{x \in E}\left\{\left\langle x, x^{*}\right\rangle-g(x)\right\}
$$

for all $x^{*} \in E^{*}$. It is well known that $g(x)+g^{*}\left(x^{*}\right) \geq\left\langle x, x^{*}\right\rangle$ for all $\left(x, x^{*}\right) \in E \times E^{*}$. It is also known that $\left(x, x^{*}\right) \in \partial g$ is equivalent to

$$
g(x)+g^{*}\left(x^{*}\right)=\left\langle x, x^{*}\right\rangle .
$$

Here, $\partial g$ is the subdifferential of $g[35,36]$. We also know that if $g: E \rightarrow(-\infty,+\infty]$ is a proper, lower semicontinuous and convex function, then $g^{*}: E^{*} \rightarrow(-\infty,+\infty]$ is a proper, weak* lower semicontinuous and convex function; see [37] for more details on convex analysis.

2.2. Some facts on uniformly convex functions. Let $E$ be a Banach space and let $B_{r}:=\{z \in$ $E:\|z\| \leq r\}$ for all $r>0$. Then a function $g: E \rightarrow \mathbb{R}$ is said to be uniformly convex on bounded subsets of $E$ ([40], pp. 203, 221) if $\rho_{r}(t)>0$ for all $r, t>0$, where $\rho_{r}:[0,+\infty) \rightarrow[0, \infty]$ is defined by

$$
\rho_{r}(t)=\inf _{x, y \in B_{r},\|x-y\|=t, \alpha \in(0,1)} \frac{\alpha g(x)+(1-\alpha) g(y)-g(\alpha x+(1-\alpha) y)}{\alpha(1-\alpha)}
$$

for all $t \geq 0$. The function $\rho_{r}$ is called the gauge of uniform convexity of $g$. The function $g$ is also said to be uniformly smooth on bounded subsets of $E$ ([40], pp. 207, 221) if $\lim _{t \downarrow 0} \frac{\sigma_{r}(t)}{t}=0$ for all $r>0$, where $\sigma_{r}:[0,+\infty) \rightarrow[0, \infty]$ is defined by

$$
\sigma_{r}(t)=\sup _{x \in B_{r}, y \in S_{E}, \alpha \in(0,1)} \frac{\alpha g(x+(1-\alpha) t y)+(1-\alpha) g(x-\alpha t y)-g(x)}{\alpha(1-\alpha)}
$$

for all $t \geq 0$. The function $g$ is said to be uniformly convex if the function $\delta_{g}:[0,+\infty) \rightarrow[0,+\infty]$, defined by

$$
\delta_{g}(t):=\sup \left\{\frac{1}{2} g(x)+\frac{1}{2} g(y)-g\left(\frac{x+y}{2}\right):\|y-x\|=t\right\},
$$

satisfies that $\lim _{t \downarrow 0} \frac{\delta_{g}(t)}{t}=0$. 
Definition 2.1. ([4]). Let $E$ be a Banach space. The function $g: E \rightarrow(-\infty,+\infty]$ is said to be a Bregman function if the following conditions are satisfied:

(1) $g$ is continuous, strictly convex and Gâteaux differentiable;

(2) the set $\left\{y \in E: D_{g}(x, y) \leq r\right\}$ is bounded for all $x \in E$ and $r>0$.

The following lemma follows from Butnariu and Iusem [4] and Zălinscu [40].

Lemma 2.1. Let $E$ be a reflexive Banach space and $g: E \rightarrow(-\infty,+\infty]$ a strongly coercive Bregman function. Then

(1) $\nabla g: E \rightarrow E^{*}$ is one-to-one, onto and norm-to-weak ${ }^{*}$ continuous;

(2) $\langle x-y, \nabla g(x)-\nabla g(y)\rangle=0$ if and only if $x=y$;

(3) $\left\{x \in E: D_{g}(x, y) \leq r\right\}$ is bounded for all $y \in E$ and $r>0$;

(4) dom $g^{*}=E^{*}, g^{*}$ is Gâteaux differentiable and $\nabla g^{*}=(\nabla g)^{-1}$.

Lemma 2.2. [27] Let $E$ be a Banach space and $g: E \rightarrow(-\infty,+\infty]$ a Gâteaux differentiable function which is uniformly convex on bounded subsets of E. Let $\left\{x_{n}\right\}_{n \in \mathbb{N}}$ and $\left\{y_{n}\right\}_{n \in \mathbb{N}}$ be bounded sequences in E. Then

$$
\lim _{n \rightarrow \infty} D_{g}\left(x_{n}, y_{n}\right)=0 \Longleftrightarrow \lim _{n \rightarrow \infty}\left\|x_{n}-y_{n}\right\|=0 .
$$

Theorem 2.1. [40] Let $E$ be a reflexive Banach space and $g: E \rightarrow(-\infty,+\infty]$ a convex function which is bounded on bounded subsets of $E$. Then the following assertions are equivalent:

(1) $g$ is strongly coercive and uniformly convex on bounded subsets of $E$;

(2) dom $g^{*}=E^{*}, g^{*}$ is bounded on bounded subsets and uniformly smooth on bounded subsets of $E^{*}$;

(3) dom $g^{*}=E^{*}, g^{*}$ is Fréchet differentiable and $\nabla g^{*}$ is uniformly norm-to-norm continuous on bounded subsets of $E^{*}$.

Theorem 2.2. [40] Let $E$ be a reflexive Banach space and $g: E \rightarrow(-\infty,+\infty]$ a continuous convex function which is strongly coercive. Then the following assertions are equivalent:

(1) $g$ is bounded on bounded subsets and uniformly smooth on bounded subsets of E;

(2) $g^{*}$ is Fréchet differentiable and $\nabla g^{*}$ is uniformly norm-to-norm continuous on bounded subsets of $E^{*}$;

(3) dom $g^{*}=E^{*}, g^{*}$ is strongly coercive and uniformly convex on bounded subsets of $E^{*}$.

At the end of this section, we include the following important lemma, which plays important roles in the sequel.

Lemma 2.3. [8] Let $E$ be a reflexive Banach space. Let $S$ be a semitopological semigroup and $h: S \rightarrow E$ be a bounded function such that $\{h(s): s \in S\}$ is bounded. Let $\mathcal{R}=\left\{T_{s}: s \in S\right\}$ be a representation of $S$ as mappings on $S$. Let $X$ be a closed subspace of $\ell^{\infty}(S)$ and $\mu$ be a mean on $X$. Then there exists a unique point $z_{0} \in \overline{c o}\{h(s): s \in S\}$ such that

$$
\mu_{s}\left\langle h(s), y^{*}\right\rangle=\left\langle z_{0}, y^{*}\right\rangle \quad \forall y^{*} \in E^{*} .
$$

Lemma 2.4. Let $E$ be a reflexive Banach space and $g: E \rightarrow(-\infty,+\infty]$ a strongly coercive Bregman function which is uniformly convex on bounded subsets of $E$. Let $C$ be a nonempty subset of $E$ and $X$ be a closed subspace of $\ell^{\infty}(S)$ containing the constant functions and being 
invariant under left translations. Let $\mathcal{R}=\left\{T_{S}: s \in S\right\}$ be a representation of a semigroup $S$ on $C$ such that $\left\{T_{s} x: s \in S\right\}$ is bounded for some $x \in C$ and

$$
D_{g}\left(T_{s} x, T_{t} y\right) \leq D_{g}\left(T_{s} x, y\right)
$$

for all $y \in C, t \in S$. If $X$ has a left invariant mean $\mu$, then $T_{\mu} x \in A_{C}^{g}(\mathcal{R})$ and so $A_{C}^{g}(\mathcal{R}) \neq \emptyset$, where $T_{\mu} x$ is as in Lemma 2.3. In particular, if $C$ is closed and convex, then $F(\mathcal{R})=\cap\left\{F\left(T_{S}\right)\right.$ : $s \in S\} \neq \emptyset$.

Let $E$ be a reflexive Banach space and let $g: E \rightarrow(-\infty,+\infty]$ be a convex, continuous and strongly coercive function which is bounded on bounded sets, and uniformly convex and uniformly smooth on bounded sets. Let $C_{*}$ be a nonempty closed convex subset of $E^{*}$ and let proj ${ }_{C_{*}}^{g}$ be the Bregman projection from $E^{*}$ onto $C_{*}$. Let $C$ be a nonempty subset of $E$ and $T: C \rightarrow E$ be a mapping. We define the duality mapping $T^{*}: \nabla g C \rightarrow E^{*}$ of $T$ by

$$
T^{*} x^{*}=\nabla g T(\nabla g)^{-1} x^{*} \quad \forall x^{*} \in \nabla g C,
$$

where $\nabla g$ is the duality mapping on $E$ and $(\nabla g)^{-1}$ is the duality mapping on $E^{*}$. The mapping $T^{*}$ is called the Bregman duality mapping of $T$; see [25].

Let $E$ be a Banach space and let $g: E \rightarrow(-\infty,+\infty]$ be a convex and Gâteaux differentiable function. Let $C$ be a nonempty and closed subset of $E$. A mapping $T: C \rightarrow E$ is called Bregman generalized nonexpansive if $F(T) \neq \varnothing$ and

$$
D_{g}(T x, p) \leq D_{g}(x, p), \forall(x, p) \in C \times F(T) .
$$

Let $C$ be a nonempty subset of real Banach space $E$. A mapping $R: E \rightarrow C$ is said to be sunny if

$$
R(R x+t(x-R x))=R x
$$

for each $x \in E$ and $t \geq 0$. A mapping $R: E \rightarrow C$ is said to be a retraction if $R x=x$ for each $x \in C$, for more details, see [25].

Lemma 2.5. ([26]) Let $E$ be a reflexive Banach space and let $g: E \rightarrow(-\infty,+\infty]$ be a strongly coercive Bregman function. Let $C$ be a nonempty closed subset of $E$ and let $R_{C}^{g}$ be a retraction from $E$ onto $C$. Then the following assertions are equivalent:

(1) $R_{C}^{g}$ is sunny and Bregman generalized nonexpansive;

(2) $\left\langle x-R_{C}^{g} x, \nabla g(y)-\nabla g\left(R_{C}^{g} x\right)\right\rangle \leq 0, \forall(x, y) \in E \times C$.

Furthermore, a sunny and Bregman generalized nonexpansive retraction of $E$ onto $C$ is uniquely determined.

Lemma 2.6. ([26]) Let $E$ be a reflexive Banach space and let $g: E \rightarrow(-\infty,+\infty]$ be a strongly coercive Bregman function. Let $C$ be a nonempty closed subset of $E$ and let $R_{C}^{g}$ be a sunny Bregman generalized nonexpansive retraction from $E$ onto $C$. Let $(x, z) \in E \times C$. Then the following assertions hold:

(1) $z=R_{C}^{g} x$ if and only if $\langle x-z, \nabla g(y)-\nabla g(z)\rangle \leq 0$ for all $y \in C$;

(2) $D_{g}\left(R_{C}^{g} x, z\right)+D_{g}\left(x, R_{C}^{g} x\right) \leq D_{g}(x, z)$.

Theorem 2.3. ([26]) Let $E$ be a reflexive Banach space and let $g: E \rightarrow(-\infty,+\infty]$ be a convex, continuous and strongly coercive function which is bounded on bounded sets, and uniformly convex and uniformly smooth on bounded sets. Let $C$ be a nonempty closed subset of $E$. Then the following statements are equivalent: 
(1) $C$ is a sunny Bregman generalized nonexpansive retract of $E$;

(2) $C$ is a Bregman generalized nonexpansive retract of $E$;

(3) $\nabla g C$ is closed and convex.

In this case, the unique sunny Bregman generalized nonexpansive retraction from $E$ onto $C$ is given by $(\nabla g)^{-1}$ proj $_{C_{*}}^{g} \nabla g$, where proj $_{C_{*}}^{g}$ is the Bregman projection from $E^{*}$ onto $\nabla g C$.

Lemma 2.7. ([26]) Let $E$ be a reflexive Banach space and let $g: E \rightarrow(-\infty,+\infty]$ be a convex, continuous and strongly coercive function which is bounded on bounded sets, and uniformly convex and uniformly smooth on bounded sets. Let $C$ be a nonempty closed subset of $E$ such that $\nabla g C$ is closed and convex. If $T: C \rightarrow C$ is a Bregman generalized nonexpansive mapping such that $F(T) \neq \varnothing$, then $F(T)$ is closed and $\nabla g F(T)$ is closed and convex.

Lemma 2.8. [25] Let $E$ be a reflexive Banach space and let $g: E \rightarrow(-\infty,+\infty]$ be a convex, continuous and strongly coercive function which is bounded on bounded sets, and uniformly convex and uniformly smooth on bounded sets. Let $C$ be a nonempty subset of $E$, let $T: C \rightarrow E$ be a mapping and $T^{*}: \nabla g C \rightarrow E^{*}$ be the duality mapping of $T$ defined in (6.1). Then the following assertions hold:

(1) $\nabla g F(T)=F\left(T^{*}\right)$;

(2) $\nabla g \hat{F}(T)=\breve{F}\left(T^{*}\right)$;

(3) $\nabla g \breve{F}(T)=\hat{F}\left(T^{*}\right)$.

Theorem 2.4. [25] Let $E$ be a reflexive Banach space and let $g: E \rightarrow(-\infty,+\infty]$ be a convex, continuous and strongly coercive function which is bounded on bounded sets, and uniformly convex and uniformly smooth on bounded sets. Let $C$ be a nonempty subset of $E$, let $T: C \rightarrow E$ be a Bregman relatively nonexpansive mapping and $T^{*}: \nabla g C \rightarrow E^{*}$ be the duality mapping of $T$ defined in (3.1). Then $T^{*}$ is Bregman generalized nonexpansive and $\breve{F}\left(T^{*}\right)=F\left(T^{*}\right)$.

\section{BREGMAN SKEW-ATTRACTIVE POINTS IN A BANACH SPACE}

In section, we investigate the Bregman Skew-attractive point theorems in a reflexive Banach space $E$.

Let $E$ be a reflexive Banach space and let $g: E \rightarrow(-\infty,+\infty]$ be a convex, continuous and strongly coercive function. Let $C$ be a nonempty subset of $E$ and $T: C \rightarrow E$ be mapping. We denote by $B_{C}^{g}(T)$ the set of Bregman skew-attractive points of $T$; i.e.,

$$
B_{C}^{g}(T)=\left\{z \in E: D_{g}(T x, x) \leq D_{g}(x, z) \quad \forall x \in C\right\} .
$$

In this section, we prove Bregman Skew-attractive point theorems in a reflexive Banach space. We start with the following simple lemma.

Lemma 3.1. Let $E$ be a reflexive Banach space and $g: E \rightarrow(-\infty,+\infty]$ a convex, continuous, strongly coercive and Gâteaux differentiable function which is bounded on bounded subsets and uniformly convex on bounded subsets of $E$. Let $C$ be a nonempty subset of $E$ and $T: C \rightarrow E$ be a mapping. Then $B_{C}^{g}(T)$ is closed and convex.

Proof. Let $\left\{y_{n}\right\}_{n \in \mathbb{N}} \subset B_{C}^{g}(T)$ be such that $y_{n} \rightarrow y$ as $n \rightarrow \infty$. Then

$$
D_{g}\left(T x, y_{n}\right) \leq D_{g}\left(x, y_{n}\right), \quad \forall x \in C, n \in \mathbb{N} .
$$


This means that

$$
g(T x)-g\left(y_{n}\right)-\left\langle T x-y_{n}, \nabla g\left(y_{n}\right)\right\rangle \leq g(x)-g\left(y_{n}\right)-\left\langle x-y_{n}, \nabla g\left(y_{n}\right)\right\rangle .
$$

Since $g$ is continuous and $\nabla g$ is norm-to-weak* continuous, we obtain

$$
g(T x)-g(y)-\langle T x-y, \nabla g(y)\rangle \leq g(x)-g(y)-\langle x-y, \nabla g(y)\rangle
$$

and hence

$$
D_{g}(T x, y) \leq D_{g}(x, y), \quad \forall x \in C .
$$

Thus we have $y \in B_{C}^{g}(T)$ which shows that $B_{C}^{g}(T)$ is closed. A similar argument, as in the proof of Lemma 3.2 of [8], demonstrates that $B_{C}^{g}(T)$ is convex, which completes the proof.

Proposition 3.1. Let $E$ be a reflexive Banach space and let $g: E \rightarrow(-\infty,+\infty]$ be a convex, continuous and strongly coercive function, which is bounded on bounded sets, and uniformly convex and uniformly smooth on bounded sets. Let $C$ be a nonempty subset of $E$, let $T: C \rightarrow E$ be a mapping and let $T^{*}: \nabla g C \rightarrow E^{*}$ be the duality mapping of $T$ defined in (2.1). Then the following assertions hold:

(1) $\nabla g B_{C}^{g}(T)=A_{C}^{g}\left(T^{*}\right)$;

(2) $\nabla g A_{C}^{g}(T)=B_{C}^{g}\left(T^{*}\right)$.

In particular, $\nabla g B_{C}^{g}(T)$ is closed and convex.

Proof. Let us prove (1). For any $x^{*} \in E^{*}$, we get from the definition of $T^{*}$ that

$$
\begin{aligned}
x^{*} \in \nabla g B_{C}^{g}(T) & \Longleftrightarrow \nabla g^{*} x^{*} \in B_{C}^{g}(T) \\
& \Longleftrightarrow D_{g}\left(T x, \nabla g^{*} x^{*}\right) \leq D_{g}\left(x, \nabla g^{*} x^{*}\right), \forall x \in C \\
& \Longleftrightarrow D_{g^{*}}\left(\nabla g^{*} x^{*}, \nabla g T x\right) \leq D_{g^{*}}\left(x^{*}, \nabla g x\right), \forall x \in C \\
& \Longleftrightarrow D_{g^{*}}\left(\nabla g^{*} x^{*}, \nabla g T \nabla g^{*} \nabla g x\right) \leq D_{g^{*}}\left(x^{*}, \nabla g x\right), \forall x \in C \\
& \Longleftrightarrow D_{g^{*}}\left(\nabla g^{*} x^{*}, T^{*} \nabla g x\right) \leq D_{g^{*}}\left(x^{*}, \nabla g x\right), \forall x \in C \\
& \Longleftrightarrow x^{*} \in \nabla g A_{C}^{g}\left(T^{*}\right) .
\end{aligned}
$$

This proves that $\nabla g B_{C}^{g}(T)=A_{C}^{g}\left(T^{*}\right)$.

Now, we prove (2) in a similar fashion. For any $x^{*} \in E^{*}$, we get from the definition of $T^{*}$ that

$$
\begin{aligned}
x^{*} \in \nabla g A_{C}^{g}(T) & \Longleftrightarrow \nabla g^{*} x^{*} \in A_{C}^{g}(T) \\
& \Longleftrightarrow D_{g}\left(\nabla g^{*} x^{*}, T x\right) \leq D_{g}\left(\nabla g^{*} x^{*}, x\right), \forall x \in C \\
& \Longleftrightarrow D_{g^{*}}\left(\nabla g T x, \nabla g^{*} x^{*}\right) \leq D_{g^{*}}\left(\nabla g x, x^{*}\right), \forall x \in C \\
& \Longleftrightarrow D_{g^{*}}\left(\nabla g T \nabla g^{*} \nabla g x, \nabla g^{*} x^{*}\right) \leq D_{g^{*}}\left(\nabla g x, x^{*}\right), \forall x \in C \\
& \Longleftrightarrow D_{g^{*}}\left(T^{*} \nabla g x, \nabla g^{*} x^{*}\right) \leq D_{g^{*}}\left(\nabla g x, x^{*}\right), \forall x \in C \\
& \Longleftrightarrow x^{*} \in \nabla g B_{C}^{g}\left(T^{*}\right) .
\end{aligned}
$$

This proves $\nabla g A_{C}^{g}(T)=B_{C}^{g}\left(T^{*}\right)$. On the other hand, in view of Lemma 3.1, we conclude that $\nabla g A_{C}^{g}(T)$ is closed and convex, so we deduce that $\nabla g A_{C}^{g}(T)$ is closed and convex. This completes the proof.

Theorem 3.1. Let $E$ be a reflexive Banach space and $g: E \rightarrow(-\infty,+\infty]$ a strongly coercive Bregman function, which is bounded on bounded subsets, and uniformly convex and uniformly smooth on bounded subsets of $E$. Let $C$ be a nonempty subset of $E$ and $X$ be a closed subspace of $\ell^{\infty}(S)$ containing the constant functions and being invariant under left translations. Let 
$\mathcal{R}=\left\{T_{s}: s \in S\right\}$ be a representation of a semigroup $S$ as mappings of $C$ into itself such that $\left\{T_{S}(x)\right\}_{s \in S}$ is bounded for some $x \in C$. Let $\mu$ be a mean on X. Suppose that

$$
D_{g}\left(T_{t} y, T_{s} x\right) \leq D_{g}\left(y, T_{s} x\right)
$$

for all $y \in C$ and $t \in S$. Then, $B_{C}^{g}(\mathcal{R})=\cap\left\{B_{C}^{g}\left(T_{t}\right): t \in S\right\}$ is nonempty. Additionally, if $C$ is closed and $\nabla g(C)$ is closed and convex, then $F(\mathcal{R})=\cap\left\{F\left(T_{t}\right): t \in S\right\}$ is nonempty.

Proof. Assume that $\left\{T_{S}(x)\right\}_{s \in S}$ is bounded for some $x \in C$. Put $x^{*}=\nabla g(x)$ and $y^{*}=\nabla g(y)$, where $y \in C$ and define

$$
T_{S}^{*}=\nabla g T_{S}(\nabla g)^{-1}, \quad \forall s \in S
$$

Then, we have

$$
T_{s}^{*} T_{t}^{*}=\nabla g T_{s}(\nabla g)^{-1} \nabla g T_{S}(\nabla g)^{-1}=\nabla g T_{s+t}(\nabla g)^{-1} \nabla g T_{s}(\nabla g)^{-1}=T_{s+t}^{*}, \quad \forall s, t \in S .
$$

On the other hand, if $s \rightarrow t$, then we have from the continuity of $\nabla g$ that, for any $y^{*} \in \nabla g(C)$,

$$
\left\|T_{s}^{*} y^{*}-T_{t}^{*} y^{*}\right\|=\left\|\nabla g T_{s}(\nabla g)^{-1} \nabla g y-\nabla g T_{t}(\nabla g)^{-1} \nabla g y\right\|=\left\|\nabla g T_{s} \nabla g y-\nabla g T_{t} \nabla g y\right\| \rightarrow 0 .
$$

Therefore, $\mathcal{R}^{*}=\left\{T_{s}^{*}: s \in S\right\}$ is a continuous representation of $S$ as mappings of $\nabla g(C)$ into itself. Furthermore, since $\left\{T_{S}(x): s \in S\right\}$ is bounded and

$$
\mu_{s} D_{g}\left(T_{t} y, T_{s} x\right) \leq \mu_{s} D_{g}\left(y, T_{s} x\right)
$$

for all $y \in C$ and $t \in S$, we have

$$
\begin{aligned}
\mu_{s} D_{g^{*}}\left(T_{s}^{*} x^{*}, T_{t}^{*} y^{*}\right) & =\mu_{s} D_{g^{*}}\left(\nabla g T_{s}(\nabla g)^{-1} \nabla g x, \nabla g T_{s}(\nabla g)^{-1} \nabla g y\right) \\
& =\mu_{s} D_{g}\left(T_{t}(\nabla g)^{-1} \nabla g y, T_{s}(\nabla g)^{-1} \nabla g x\right) \\
& =\mu_{s} D_{g}\left(T_{t} y, T_{s} x\right) \\
& \leq \mu_{s} D_{g}\left(y, T_{s} x\right) \\
& =\mu_{s} D_{g^{*}}\left(\nabla g T_{s} x, \nabla g y\right) \\
& =\mu_{s} D_{g^{*}}\left(\nabla g T_{s}(\nabla g)^{-1} \nabla g x, \nabla g y\right) \\
& =\mu_{s} D_{g^{*}}\left(\nabla g T_{s}^{*} x^{*}, y^{*}\right)
\end{aligned}
$$

for all $y^{*} \in \nabla g(C)$ and $t \in S$. Therefore, we have from Lemma 2.4 that

$$
A_{C}^{g}\left(\mathcal{R}^{*}\right)=\cap\left\{A_{C}^{g}\left(T_{t}^{*}\right): t \in S\right\}
$$

is nonempty. Since $\nabla g: E \rightarrow E^{*}$ is a one-to-one and onto mapping, we obtain from Lemma 2.8 that

$$
\begin{aligned}
B_{C}^{g}(\mathcal{R}) & =\cap\left\{B_{C}^{g}\left(T_{t}\right): t \in S\right\} \\
& =\cap\left\{(\nabla g)^{-1} A_{C}^{g}\left(T_{t}^{*}\right): t \in S\right\} \\
& =(\nabla g)^{-1}\left(\cap\left\{A_{C}^{g}\left(T_{t}^{*}\right): t \in S\right\}\right) \\
& =(\nabla g)^{-1} A_{C}^{g}\left(\mathcal{R}^{*}\right) .
\end{aligned}
$$

Since $A_{C}^{g}\left(\mathcal{R}^{*}\right)$ is nonempty, we get that $B_{C}^{g}(\mathcal{R})$ is nonempty. In particular, if $C$ is closed and $\nabla g(C)$ is closed and convex, then we obtain from Lemma 2.8 that

$$
\begin{aligned}
F(\mathcal{R}) & =\cap\left\{F\left(T_{t}\right): t \in S\right\} \\
& \left.=\cap\left\{(\nabla g)^{-1} F\left(T_{t}^{*}\right): t \in S\right\}\right) \\
& =(\nabla g)^{-1}\left(\cap\left\{F\left(T_{t}^{*}\right): t \in S\right\}\right) \\
& =(\nabla g)^{-1} F\left(\mathcal{S}^{*}\right) .
\end{aligned}
$$

Thus we have $F(\mathcal{R})=\bigcap\left\{F\left(T_{t}\right): t \in S\right\}$ is nonempty, which completes the proof. 
Remark 3.1. The main result of [20] gave a relation between common attractive points and common fixed points for a semigroup of nonexpansive mappings on a closed convex subset $C$ of a Hilbert space, while the main result of the present paper give a relation between common fixed points and Bregman common skew-attractive points for a semigroup of Bregman nonexpansive type (not necessarily continuous) mappings on a closed convex subset $C$ of a reflexive Banach space $E$. We note that the proof of Theorem 3.1 (where the authors used the nonexpansivity of the mapping $T$ ) in [20] is not valid in our discussion. So our result extends and improves the corresponding results of [20].

Remark 3.2. Theorem 3.1 improves [20, Theorem 3.1] in the following aspects.

(1) For the structure of Banach spaces, we extend the duality mapping to more general case, that is, a convex, continuous and strongly coercive Bregman function which is bounded on bounded subsets, and uniformly convex and uniformly smooth on bounded subsets.

(2) For the mappings, we extend the mapping from nonexpansive mappings to Bregman nonexpansive type (not necessarily continuous) mappings.

\section{BREGMAN COMMON SKEW-ATTRACTIVE POINT THEOREMS}

In this section, we prove Bregman common skew-attractive point theorems of Baillon's type [1] for semigroups of nonlinear mappings without continuity in a Banach space. We first prove the following important lemma.

Lemma 4.1. Let $E$ be a reflexive Banach space and $g: E \rightarrow(-\infty,+\infty]$ a strongly coercive Bregman function, which is bounded on bounded subsets, and uniformly convex and uniformly smooth on bounded subsets of $E$. Let $S$ be a semigroup, $D$ be a nonempty subset of $E$ and let $X$ be a closed subspace of $\ell^{\infty}(S)$ containing the constant functions and being invariant under left translations. Let $\mathcal{R}=\left\{T_{S}: s \in S\right\}$ be a representation of $S$ as mappings of $D$ into itself such that $\left\{T_{S}(x)\right\}_{s \in S} \subset D$ is bounded for some $x \in D$. Let $v: S \rightarrow E$ be a function such that $\{v(s): s \in S\} \subset D$ is bounded and let $\mu$ be a mean on $X$. If $h: D \rightarrow \mathbb{R}$ is defined by

$$
h(z)=\mu_{s} D_{g}(v(s), z) \quad \forall z \in D,
$$

then the mean vector $z_{0}$ of $\{v(s): s \in S\}$ for $\mu$ is a unique minimizer in $D$ such that

$$
h\left(z_{0}\right)=\min \{h(z): z \in D\} .
$$

Proof. For a bounded net $\{v(s): s \in S\} \subset D$ and a mean $\mu$ on $X$, we know that a function $h: D \rightarrow \mathbb{R}$ defined by

$$
h(z)=\mu_{s} D_{g}(v(s), z) \quad \forall z \in D
$$

is well-defined. We also know from the proof of Lemma 2.3 that there exists a mean vector $z_{0}$ of $\{v(s): s \in S\}$ for $\mu$, that is, there exists $z_{0} \in \overline{c o}\{v(s): s \in S\}$ such that

$$
\mu_{s}\left\langle v(s), y^{*}\right\rangle=\left\langle z_{0}, y^{*}\right\rangle \quad \forall y^{*} \in E^{*}
$$


Since $D$ is closed and convex and $\{v(s): s \in S\} \subset D$, we get $z_{0} \in D$. Furthermore, we obtain from the three-point identity (see (1.3)) that, for any $z \in D$, that

$$
\begin{aligned}
h(z)-h\left(z_{0}\right) & =\mu_{s} D_{g}(v(s), z)-\mu_{s} D_{g}\left(v(s), z_{0}\right) \\
& =\mu_{s}\left(D_{g}(v(s), z)-D_{g}\left(v(s), z_{0}\right)\right) \\
& =\mu_{s}\left(D_{g}(v(s), z)-D_{g}(v(s), z)-D_{g}\left(z, z_{0}\right)-\left\langle v(s)-z, \nabla g(z)-\nabla g\left(z_{0}\right)\right\rangle\right) \\
& =\mu_{s}\left(-D_{g}\left(z, z_{0}\right)-\left\langle v(s)-z, \nabla g(z)-\nabla g\left(z_{0}\right)\right\rangle\right) \\
& =\mu_{s}\left(-D_{g}\left(z, z_{0}\right)-\left\langle v(s)-z, \nabla g(z)-\nabla g\left(z_{0}\right)\right\rangle\right) \\
& =-D_{g}\left(z, z_{0}\right)-\left\langle v(s)-z, \nabla g(z)-\nabla g\left(z_{0}\right)\right\rangle \\
& =-D_{g}\left(z, z_{0}\right)-D_{g}\left(z_{0}, z_{0}\right)-D_{g}(z, z)-D_{g}\left(z_{0}, z\right)-D_{g}\left(z, z_{0}\right) \\
& =D_{g}\left(z_{0}, z\right) .
\end{aligned}
$$

Thus we have

$$
h(z)=h\left(z_{0}\right)+D_{g}\left(z_{0}, z\right), \quad \forall z \in D .
$$

This implies that $z_{0} \in D$ is a minimizer in $D$ such that

$$
h\left(z_{0}\right)=\min \{h(z): z \in D\} .
$$

Furthermore, if $v \in D$ satisfies $h(v)=h\left(z_{0}\right)$, then we infer from (4.1) that $D_{g}\left(z_{0}, v\right)=0$. This, together with Lemma 2.2, implies that $z_{0}=v$ and hence $z_{0}$ is a unique minimizer in $D$ such that $h\left(z_{0}\right)=\min \{h(z): z \in D\}$. This completes the proof.

Theorem 4.1. Let $E$ be a reflexive Banach space and $g: E \rightarrow(-\infty,+\infty]$ a strongly coercive Bregman function which is bounded on bounded subsets, and uniformly convex and uniformly smooth on bounded subsets of $E$. Let $S$ be a semigroup, $C$ be a nonempty, closed and convex subset of $E$ and $X$ be a closed subspace of $\ell^{\infty}(S)$ containing the constant functions and being invariant under left translations. Let $\mathcal{R}=\left\{T_{s}: s \in S\right\}$ be a representation of $S$ as mappings of $C$ into itself such that $\left\{T_{S}\left(x_{0}\right)\right\}_{s \in S} \subset C$ is bounded for some $x_{0} \in C$. Suppose that $A_{C}^{g}(\mathcal{R})=B_{C}^{g}(\mathcal{R})$ is nonempty. Then, for any $x \in C$, the net $\mathcal{R} x=\left\{T_{s} x: s \in S\right\}$ is bounded and the set

$$
\cap_{s \in S} \overline{c o}\left\{T_{t+s} x: t \in S\right\} \cap A_{C}^{g}(\mathcal{R})
$$

consists of one point $z_{0}$, where $z_{0}$ is a unique minimizer of $A_{C}^{g}(\mathcal{R})$ such that

$$
\lim _{s \in S} D_{g}\left(T_{s} x, z_{0}\right)=\min \left\{\lim _{s \in S} D_{g}\left(T_{s} x, z\right): z \in A_{C}^{g}(\mathcal{R})\right\} .
$$

Additionally, if $C$ is closed and convex, then the set

$$
\cap_{s \in S} \overline{c o}\left\{T_{t+s} x: t \in S\right\} \cap F(\mathcal{R})
$$

consists of one point $z_{0}$.

Proof. Since $A_{C}^{g}(\mathcal{R})=B_{C}^{g}(\mathcal{R})$ is nonempty, then, for any $z \in A_{C}^{g}(\mathcal{R})=B_{C}^{g}(\mathcal{R})$ and $x \in C$, we obtain

$$
D_{g}\left(T_{t+s} x, z\right) \leq D_{g}\left(T_{s} x, z\right), \quad \forall s, t \in S .
$$

This implies that the set $\mathcal{R} x=\left\{T_{s} x: s \in S\right\}$ is bounded. For any invariant mean $\mu$ on $X$, in view of Lemma 4.1, there exists a unique minimizer $z_{0} \in E$ such that

$$
\mu_{s} D_{g}\left(T_{s} x, z_{0}\right)=\min \left\{\mu_{s} D_{g}\left(T_{s} x, y\right): y \in E\right\}
$$

is the mean vector $z_{0} \in E$ of $\mathcal{R}=\left\{T_{s}: s \in S\right\}$ for $\mu$, that is, a point $z_{0} \in E$ such that $z_{0} \in$ $\cap_{s \in S} \overline{c o}\left\{T_{s} x: s \in S\right\}$ and

$$
\mu_{s}\left\langle T_{s} x, y^{*}\right\rangle=\left\langle z_{0}, y^{*}\right\rangle, \quad y^{*} \in E^{*} .
$$


By the same arguments, as in the proof of Lemma 2.4, we conclude that $z_{0} \in A_{C}^{g}(\mathcal{R})$ and at the same time $z_{0} \in A_{C}^{g}(\mathcal{R})$ satisfies

$$
\mu_{s} D_{g}\left(T_{s} x, z_{0}\right)=\min \left\{\mu_{s} D_{g}\left(T_{s} x, z\right): z \in A_{C}^{g}(\mathcal{R})\right\} .
$$

We claim that $z_{0} \in \cap_{s \in S} \overline{c o}\left\{T_{t+s} x: t \in S\right\}$. If not, then there exists $s_{0} \in S$ such that $z_{0} \notin$ $\cap_{s \in S} \overline{c o}\left\{T_{t+s_{0}} x: t \in S\right\}$. In view of the separation theorem (a well-known corollary of the HahnBanach theorem), there exists $y_{0}^{*} \in E^{*}$ such that

$$
\left\langle z_{0}, y_{0}^{*}\right\rangle<\inf \left\{\left\langle z, y_{0}^{*}\right\rangle: z \in \overline{c o}\left\{T_{t+s_{0}} x: t \in S\right\}\right\} .
$$

By the invariance of $\mu$, we get

$$
\begin{aligned}
\left\langle z_{0}, y_{0}^{*}\right\rangle & <\inf \left\{\left\langle z, y_{0}^{*}\right\rangle: z \in \overline{c o}\left\{T_{t+s_{0}} x: t \in S\right\}\right\} \\
& \left.\leq \inf \left\{\left\langle T_{t+s_{0}} x, y_{0}^{*}\right\rangle: t \in S\right\}\right\} \\
& \leq \mu_{t}\left\langle T_{t+s_{0}} x, y_{0}^{*}\right\rangle \\
& =\mu_{t}\left\langle T_{t} x, y_{0}^{*}\right\rangle \\
& =\left\langle z_{0}, y_{0}^{*}\right\rangle .
\end{aligned}
$$

This is a contradiction. Therefore, $z_{0} \in \cap_{s \in S} \overline{c o}\left\{T_{t+s} x: t \in S\right\}$. We still intend to show that $\cap_{s \in S} \overline{C O}\left\{T_{t+s} x: t \in S\right\} \cap A_{C}^{g}(\mathcal{R})$ consists of exactly one point $z_{0}$. Assume that

$$
z_{1} \in \cap_{s \in S} \overline{c o}\left\{T_{t+s} x: t \in S\right\} \cap A_{C}^{g}(\mathcal{R}) .
$$

Since $z_{1} \in A_{C}^{g}(\mathcal{R})=B_{C}^{g}(\mathcal{R})$, we obtain

$$
D_{g}\left(T_{t+s} x, z_{1}\right) \leq D_{g}\left(T_{s} x, z_{1}\right), \quad \forall s, t \in S,
$$

which implies that the $\operatorname{limit}_{\lim _{s}} D_{g}\left(T_{s} x, z_{1}\right)$ exists. On the other hand, the invariance property of $\mu$ leads us to

$$
\mu_{s} D_{g}\left(T_{s} x, z_{1}\right)=\lim _{s} D_{g}\left(T_{s} x, z_{1}\right) .
$$

This allows us to conclude that $\lim _{s} D_{g}\left(T_{s} x, z\right)$ exists for every $z \in A_{C}^{g}(\mathcal{R})$, so we can define a function $h: A_{C}^{g}(\mathcal{R}) \rightarrow \mathbb{R}$ as follows:

$$
h(z)=\lim _{s} D_{g}\left(T_{s} x, z\right), \quad \forall z \in A_{C}^{g}(\mathcal{R}) .
$$

In view of the three-point identity (see (1.3)), we deduce that

$$
D_{g}\left(z_{0}, z_{1}\right)=D_{g}\left(T_{s} x, z_{1}\right)-D_{g}\left(T_{s} x, z_{0}\right)-\left\langle T_{s} x-z_{0}, \nabla g\left(z_{0}\right)-\nabla g\left(z_{1}\right)\right\rangle
$$

for every $s \in S$. This entails to

$$
\begin{aligned}
D_{g}\left(z_{0}, z_{1}\right) & +\lim _{s}\left\langle T_{s} x-z_{0}, \nabla g\left(z_{0}\right)-\nabla g\left(z_{1}\right)\right\rangle \\
& =\lim _{s} D_{g}\left(T_{s} x, z_{1}\right)-\lim _{s} D_{g}\left(T_{s} x, z_{0}\right) \\
& \geq 0 .
\end{aligned}
$$

Now, for every $\varepsilon>0$, we arrive at

$$
\lim _{s}\left\langle T_{s} x-z_{0}, \nabla g\left(z_{0}\right)-\nabla g\left(z_{1}\right)\right\rangle>-D_{g}\left(z_{0}, z_{1}\right)-\varepsilon
$$

which implies that there exists $s_{1} \in S$ such that

$$
\left\langle T_{s+s_{1}} x-z_{0}, \nabla g\left(z_{0}\right)-\nabla g\left(z_{1}\right)\right\rangle>-D_{g}\left(z_{0}, z_{1}\right)-\varepsilon, \quad \forall s \in S .
$$

Since $z_{1} \in \cap_{s \in S} \overline{c o}\left\{T_{t+s} x: t \in S\right\}$, we get

$$
\left\langle z_{1}-z_{0}, \nabla g\left(z_{0}\right)-\nabla g\left(z_{1}\right)\right\rangle>-D_{g}\left(z_{0}, z_{1}\right)-\varepsilon .
$$


Taking into account the three-point identity (see (1.3)), we infer that

$$
D_{g}\left(z_{1}, z_{1}\right)+D_{g}\left(z_{0}, z_{0}\right)-D_{g}\left(z_{1}, z_{0}\right)-D_{g}\left(z_{0}, z_{1}\right) \geq-D_{g}\left(z_{0}, z_{1}\right)-\varepsilon
$$

and hence $D_{g}\left(z_{1}, z_{0}\right) \leq \varepsilon$ from which we conclude that $D_{g}\left(z_{1}, z_{0}\right)=0$. In view of Lemma 2.2, we deduce that $z_{1}=z_{0}$. Thus we have

$$
z_{0} \in \cap_{s \in S} \overline{c o}\left\{T_{t+s} x: t \in S\right\} \cap F(\mathcal{R}) .
$$

Additionally, if $C$ is closed and convex, then

$$
\cap_{s \in S} \overline{c o}\left\{T_{t+s} x: t \in S\right\} \cap A_{C}^{g}(\mathcal{R})=\left\{z_{0}\right\} .
$$

Since $\cap_{s \in S} \overline{c o}\left\{T_{t+s} x: t \in S\right\} \cap A_{C}^{g}(\mathcal{S})$ consists of one point $z_{0}$, we have

$$
\cap_{s \in S} \overline{c o}\left\{T_{t+s} x: t \in S\right\} \cap F(\mathcal{R})=\left\{z_{0}\right\} .
$$

This completes the proof.

Lemma 4.2. Let $E$ be a reflexive Banach space and $g: E \rightarrow(-\infty,+\infty]$ a strongly coercive Bregman function, which is bounded on bounded subsets, and uniformly convex and uniformly smooth on bounded subsets of E. Let $S$ be a semitopological semigroup. Let D be a nonempty, closed and convex subset of $E$ and $X$ be a closed subspace of $\ell^{\infty}(S)$ containing the constant functions and being invariant under left translations. Let $\mathcal{R}=\left\{T_{S}: s \in S\right\}$ be a representation of $S$ as mappings of $C$ into itself such that $\left\{T_{S}(x)\right\}_{s \in S} \subset D$ is bounded for some $x \in C$ and

$$
D_{g}\left(T_{s} x, T_{t} y\right) \leq D_{g}\left(T_{s} x, y\right), \quad y \in C, t \in S .
$$

Let $\left\{\mu_{\alpha}\right\}_{\alpha \in I}$ be an asymptotically invariant net of means on $X$; i.e., for each $f \in X$ and $s \in S$, $\mu_{\alpha}(f)-\mu_{\alpha}\left(\ell_{s} f\right) \rightarrow 0$. If a subnet $\left\{\mu_{\alpha_{\beta}}\right\}_{\beta}$ of $\left\{\mu_{\alpha}\right\}_{\alpha \in I}$ converges weakly to a point $u \in E$, then $u \in A_{C}^{g}(\mathcal{R})$. Additionally, if $C$ is closed and convex, then $u \in F(\mathcal{R})$.

Proof. Since $\left\{\mu_{\alpha}\right\}_{\alpha \in I}$ is a net of means on $X$, it has a cluster point $\mu$ in the weak* topology. We prove that $\mu$ is an invariant mean on $X$. Indeed, since the set

$$
Q:=\left\{\lambda \in X^{*}: \lambda(e)=\|\lambda\|=1\right\}
$$

is closed in the weak* topology, it follows that $\mu$ is a mean on $X$, where $e(s)=1$ for all $s \in S$. Indeed, $S$ is a semitopological semigroup and $B(S)$ is the Banach space of all bounded realvalued functions on $\mathrm{S}$ with supremum norm. Let $C(S)$ be the Banach subspace of $B(S)$ of all bounded real valued continuous functions on $\mathrm{S}$, with dual Banach space $C(S)^{*}$ (the dual space of $C(S)$ ). By Banach-Alaoglu Theorem, the norm closed unit ball of $C(S)^{*}$ is weak* compact. Since $e$ is represented as a weak* continuous function on $S$ (by definition), the set $\left\{\lambda \in C(S)^{*}:\langle e, \lambda\rangle=\lambda(e)=1\right\}$ is weak* compact, as it is the intersection of the preimage of the closed set $\{1\}$ of the function $e$ and the norm closed unit ball. On the other hand, you can check this directly. Suppose $\lambda_{\alpha}(e)=1=\left\|\lambda_{\alpha}\right\|$ for infinitely many distinct norm one linear functionals $\lambda_{\alpha}$ of the Banach space $C(S)$. Since the norm closed unit ball is weak* compact, there is a weak* cluster point $\lambda$ of them with norm $\|\lambda\| \leq 1$. By the weak* continuity of $e$, we see that $\|\lambda\| \geq \lambda(e)=1$. This also forces $\|\lambda\|=1$. Thus the set $Q$ is weak* compact.

Furthermore, since $\left\{\mu_{\alpha}\right\}_{\alpha \in I}$ is asymptotically invariant, for any $\varepsilon>0, f \in X$ and $s \in S$, there exists $\alpha_{0} \in I$ such that

$$
\mu_{\alpha}(f)-\mu_{\alpha}\left(\ell_{s} f\right) \mid \leq \frac{\varepsilon}{3}, \quad \forall \alpha \geq \alpha_{0}
$$


Since $\mu$ is a cluster point of $\left\{\mu_{\alpha}\right\}_{\alpha \in I}$, we can chose $\beta \geq \alpha_{0}$ such that

$$
\left|\mu_{\beta}(f)-\mu(f)\right| \leq \frac{\varepsilon}{3} \quad \text { and }\left|\mu_{\beta}\left(\ell_{s} f\right)-\mu\left(\ell_{s} f\right)\right| \leq \frac{\varepsilon}{3} .
$$

This implies that

$$
\begin{aligned}
\left|\mu(f)-\mu\left(\ell_{s} f\right)\right| & \leq\left|\mu(f)-\mu\left(\ell_{s} f\right)\right|+\left|\mu_{\beta}(f)-\mu_{\beta}\left(\ell_{s} f\right)\right| \\
& +\left|\mu_{\beta}(f)-\mu\left(\ell_{s} f\right)\right| \leq \frac{\varepsilon}{3}+\frac{\varepsilon}{3}+\frac{\varepsilon}{3} .
\end{aligned}
$$

Since $\varepsilon>0$ is arbitrary, we deduce that

$$
\mu(f)=\mu\left(\ell_{s} f\right), \quad \forall f \in X, s \in S .
$$

Suppose that a subnet $\left\{T_{\mu_{\alpha_{\beta}}} x\right\}_{\beta \in I}$ of $\left\{T_{\mu_{\alpha}} x\right\}_{\alpha \in I}$ converges weakly to some $u \in E$. If $\lambda$ is also a cluster point of $\left\{\mu_{\alpha_{\beta}}\right\}_{\beta \in I}$ in the weak* topology, then $\lambda$ is a cluster point of $\left\{\mu_{\alpha}\right\}_{\alpha \in I}$. Then $\lambda$ is an invariant mean on $X$. Without loss of generality, we may assume that $\mu_{\alpha_{\beta}} \rightarrow \lambda$ in the weak ${ }^{*}$ topology. Furthermore, we obtain from $T_{\mu_{\alpha_{\beta}}} x \rightarrow u$ that

$$
\lambda_{s}\left\langle T_{s} x, y^{*}\right\rangle=\lim _{\beta}\left(\mu_{\alpha_{\beta}}\right)_{s}\left\langle T_{s} x, y^{*}\right\rangle=\lim _{\beta} \mu_{\alpha_{\beta}}\left\langle T_{s} x, y^{*}\right\rangle=\left\langle u, y^{*}\right\rangle \forall y^{*} \in E^{*} .
$$

On the other hand, we have from three-point identity that for $y \in C$ and $s, t \in S$,

$$
\left\langle T_{s} x-T_{t} y, \nabla g(y)-\nabla g\left(T_{t} y\right)\right\rangle-D_{g}\left(T_{t} y, y\right)=D_{g}\left(T_{s} y, T_{t} y\right)-D_{g}\left(T_{s} x, y\right) .
$$

Applying $\left\{\mu_{\alpha_{\beta}}\right\}_{\beta \in I}$ on both sides of (4.2), we receive

$$
\begin{aligned}
\left(\mu_{\alpha_{\beta}}\right)_{s}\left\langle T_{s} x-T_{t} y,\right. & \left.\nabla g(y)-\nabla g\left(T_{t} y\right)\right\rangle-D_{g}\left(T_{t} x, y\right) \\
& =\left(\mu_{\alpha_{\beta}}\right)_{s} D_{g}\left(T_{s} x, T_{t} y\right)-\left(\mu_{\alpha_{\beta}}\right)_{s} D_{g}\left(T_{s} x, y\right) .
\end{aligned}
$$

Since $\mu_{\alpha_{\beta}} \rightarrow \lambda$ in the weak* topology, we conclude that

$$
\left\langle T_{s} x-T_{t} y, \nabla g(y)-\nabla g\left(T_{t} y\right)\right\rangle-D_{g}\left(T_{t} x, y\right)=\lambda_{s} D_{g}\left(T_{s} x, T_{t} y\right)-\lambda_{s} D_{g}\left(T_{s} x, y\right)
$$

and hence

$$
\left\langle u-T_{t} y, \nabla g(y)-\nabla g\left(T_{y}\right)\right\rangle-D_{g}\left(T_{t} x, y\right)=\lambda_{s} D_{g}\left(T_{s} x, T_{t} y\right)-\lambda_{s} D_{g}\left(T_{s} x, y\right) .
$$

Since $\lambda_{s} D_{g}\left(T_{s} x, T_{t} y\right)-(\lambda)_{s} D_{g}\left(T_{s} x, y\right) \leq 0$ by the assumption, we get

$$
\left\langle u-T_{t} y, \nabla g(y)-\nabla g\left(T_{t} y\right)\right\rangle-D_{g}\left(T_{t} x, y\right) \leq 0 .
$$

On the other hand, we know that

$$
\left\langle u-T_{t} y, \nabla g(y)-\nabla g\left(T_{t} y\right)\right\rangle-D_{g}\left(T_{t} y, y\right)=D_{g}\left(u, T_{t} y\right)-D_{g}(u, y),
$$

which implies that

$$
D_{g}\left(u, T_{t} y\right) \leq D_{g}(u, y), \quad y \in C, t \in S .
$$

Thus we have $u \in A_{C}^{g}\left(T_{t}\right)$ and hence $u \in A_{C}^{g}(\mathcal{R})$. In particular, if $C$ is closed and convex, then $u$ is an element of $C$. Putting $y=u$ in (4.2), we receive $T_{t} u=u$, which implies that

$$
u \in F(\mathcal{R})=\cap\left\{F\left(T_{t}\right): t \in S\right\} .
$$

This completes the proof. 
Lemma 4.3. Let $E$ be a reflexive Banach space and $g: E \rightarrow(-\infty,+\infty]$ a strongly coercive Bregman function, which is bounded on bounded subsets, and uniformly convex and uniformly smooth on bounded subsets of $E$. Let $C$ be a nonempty subset of $E$ and $S$ be a semitopological semigroup. Let $X$ be a closed subspace of $\ell^{\infty}(S)$ containing the constant functions and being invariant under left translations. Let $\mathcal{R}=\left\{T_{S}: s \in S\right\}$ be a representation of $S$ as mappings of $C$ into itself such that $B_{C}^{g}(\mathcal{R}) \neq \emptyset$. Then there exists a unique sunny Bregman generalized nonexpansive retraction $R_{C}^{g}$ of $E$ onto $B_{C}^{g}(\mathcal{R})$. Furthermore, for any $x \in C, \lim _{s} R_{C}^{g} T_{s} x$ exists in $B_{C}^{g}(\mathcal{R})$, where $\lim _{s} R_{C}^{g} T_{s} x=q$ means that $\lim _{s}\left\|R_{C}^{g} T_{s} x-q\right\|=0$.

Proof. In view of Lemmas 3.1 and Proposition 3.1, we conclude that $B_{C}^{g}(\mathcal{R})$ is closed and $\nabla g B_{C}^{g}(\mathcal{R})$ is closed and convex. Theorem 2.3 and Proposition 3.1 ensure the existence of a unique sunny Bregman generalized nonexpansive retraction $R_{C}^{g}$ of $E$ onto $B_{C}^{g}(\mathcal{R})$. Thus, for any left invariant $\mu$ on $X$, there exists $q \in E$ such that

$$
\mu_{t}\left\langle R_{C}^{g} T_{t} x, y^{*}\right\rangle=\left\langle q, y^{*}\right\rangle, \quad \forall y^{*} \in E^{*},
$$

which amounts to

$$
\mu_{t}\left\langle R_{C}^{g} T_{t+s} x, y^{*}\right\rangle=\mu_{t}\left\langle R_{C}^{g} T_{t} x, y^{*}\right\rangle=\left\langle q, y^{*}\right\rangle \quad \forall s \in S, y^{*} \in E^{*} .
$$

This implies that

$$
q \in \overline{c o}\left\{R_{C}^{g} T_{t+s} x: t \in S\right\}, \quad \forall s \in S .
$$

By the properties of sunny Bregman generalized nonexpansive retraction $R_{C}^{g}$, we deduce that

$$
0 \leq\left\langle w-R_{C}^{g} w, \nabla g\left(R_{C}^{g} w\right)-\nabla g(u)\right\rangle, \quad \forall w \in E, u \in B_{C}^{g}(\mathcal{R}) .
$$

It follows from (4.4) that

$$
\begin{aligned}
0 & \leq\left\langle w-R_{C}^{g} w, \nabla g\left(R_{C}^{g} w\right)-\nabla g(u)\right\rangle \\
& =D_{g}(w, u)+D_{g}\left(R_{C}^{g} w, R_{C}^{g} w\right)-D_{g}\left(w, R_{C}^{g} w\right)-D_{g}\left(R_{C}^{g} w, u\right) \\
& =D_{g}(w, u)-D_{g}\left(w, R_{C}^{g} w\right)-D_{g}\left(R_{C}^{g} w, u\right)
\end{aligned}
$$

which in turn implies that

$$
D_{g}\left(R_{C}^{g} w, u\right) \leq D_{g}(w, u)-D_{g}\left(w, R_{C}^{g} w\right) \quad w \in E, u \in B_{C}^{g}(\mathcal{R}) .
$$

Since $D_{g}\left(T_{s} z, u\right) \leq D_{g}(z, u)$ for all $s \in S, u \in B_{C}^{g}(\mathcal{R})$ and $z \in C$, we obtain

$$
D_{g}\left(T_{t+s} x, R_{C}^{g} T_{t+s} x\right) \leq D_{g}\left(T_{t+s} x, R_{C}^{g} T_{s} x\right) \leq D_{g}\left(T_{s} x, R_{C}^{g} T_{s} x\right), \quad y \in C, t \in S .
$$

Employing Theorem 3.1 together with (4.4), we conclude that

$$
\lim _{s} D_{g}\left(T_{s} x, R_{C}^{g} T_{s} x\right)=\inf _{r \in S} D_{g}\left(T_{r} x, R_{C}^{g} T_{r} x\right) .
$$

Setting $u=R_{C}^{g} T_{s} x$ and $w=R_{C}^{g} T_{t+s} x$ in (4.5), we arrive at

$$
\begin{aligned}
D_{g}\left(T_{t+s} x, R_{C}^{g} T_{s} x\right) & \leq D_{g}\left(T_{t+s} x, R_{C}^{g} T_{s} x\right)-D_{g}\left(T_{t+s} x, R_{C}^{g} T_{t+s} x\right) \\
& \leq D_{g}\left(T_{s} x, R_{C}^{g} T_{s} x\right)-D_{g}\left(T_{t+s} x, R_{C}^{g} T_{t+s} x\right) \\
& \leq D_{g}\left(T_{s} x, R_{C}^{g} T_{s} x\right)-\inf _{r \in S} D_{g}\left(T_{r} x, R_{C}^{g} T_{r} x\right) .
\end{aligned}
$$

Since $D_{g}(., z)$ is weakly lower semicountinuous for all $z \in E$, we obtain from (4.3) that

$$
D_{g}\left(q, R_{C}^{g} T_{s} x\right) \leq D_{g}\left(T_{s} x, R_{C}^{g} T_{s} x\right)-\inf _{r \in S} D_{g}\left(T_{r} x, R_{C}^{g} T_{r} x\right), \quad \forall s \in S .
$$

In view of (4.4), we deduce that

$$
\lim _{s}\left\|R_{C}^{g} T_{s} x-q\right\|=0
$$


Since $B_{C}^{g}(\mathcal{R})$ is closed, we see that $\left\{R_{C}^{g} T_{s} x\right\}_{s \in S}$ converges strongly to $q \in B_{C}^{g}(\mathcal{R})$, which completes the proof.

Theorem 4.2. Let $E$ be a reflexive Banach space and $g: E \rightarrow(-\infty,+\infty]$ a strongly coercive Bregman function which is bounded on bounded subsets, and uniformly convex and uniformly smooth on bounded subsets of $E$. Let $C$ be a nonempty subset of $E$ and $S$ be a semitopological semigroup. Let $X$ be a closed subspace of $\ell^{\infty}(S)$ containing the constant functions and being invariant under left translations. Let $\mathcal{R}=\left\{T_{S}: s \in S\right\}$ be a representation of $S$ as mappings of $C$ into itself such that $A_{C}^{g}(\mathcal{R})=B_{C}^{g}(\mathcal{R}) \neq \emptyset$. Let $R_{B_{C}^{g}(\mathcal{R})}$ be the sunny Bregman generalized nonexpansive retraction of $E$ onto $B_{C}^{g}(\mathcal{R})$. Suppose that

$$
D_{g}\left(T_{s} x, T_{t} y\right) \leq D_{g}\left(T_{s} x, y\right) \quad \forall x, y \in C, t \in S .
$$

for all $y \in C, t \in S$. Let $\mu$ be a left invariant mean on $X$ and let $\left\{\mu_{\alpha}\right\}_{\alpha}$ be an asymptotically invariant net of means $\mu$ on $X$; i.e., for each $f \in X$ and $s \in S$,

$$
\mu_{\alpha}(f)-\mu_{\alpha}\left(\ell_{s} f\right) \rightarrow 0
$$

Then, $\left\{T_{\mu_{\alpha}} x\right\}_{\alpha}$ converges weakly to a point $u \in A_{C}^{g}(\mathcal{R})$, where

$$
u=\lim _{s} R_{B_{C}^{g}(\mathcal{R})} T_{s} x .
$$

Additionally, if $C$ is closed and convex, then $u \in F(\mathcal{R})$, where

$$
u=\lim _{s} R_{F(\mathcal{R})}^{g} T_{s} x .
$$

Proof. Let $x \in C$ be given. Since $A_{C}^{g}(\mathcal{R})$ is nonempty, the net $\left\{T_{s} x\right\}_{s \in S}$ is bounded. By the same arguments, as in the proof of Theorem 6.5 of [38], $\left\{T_{\mu_{\alpha}} x\right\}_{\alpha \in I}$ is bounded. On the other hand, we get from Theorem 4.1 that the set

$$
\cap_{s \in S} \overline{c o}\left\{T_{t+s} x: t \in S\right\} \cap A_{C}^{g}(\mathcal{R})
$$

consists of exactly one point. In order to prove that $\left\{T_{\mu_{\alpha}} x\right\}_{\alpha \in I}$ converges weakly to a point $z_{0}$ in $A_{C}^{g}(\mathcal{R})$, it suffices to show that if a subnet $\left\{T_{\mu_{\alpha_{\beta}}} x\right\}_{\beta \in I}$ of $\left\{T_{\mu_{\alpha}}\right\}_{\alpha \in I}$ converges weakly to a point $v \in E$, i.e., $T_{\mu_{\alpha_{\beta}}} \rightarrow v$, then $v \in A_{C}^{g}(\mathcal{R})$ and

$$
v \in \cap_{s \in S} \overline{c o}\left\{T_{t+s} x: t \in S\right\} \cap A_{C}^{g}(\mathcal{R}) .
$$

In view of Lemma 4.2, we deduce that $v \in A_{C}^{g}(\mathcal{R})$. Let us show that

$$
v \in \cap_{s \in S} \overline{c o}\left\{T_{t+s} x: t \in S\right\} \cap A_{C}^{g}(\mathcal{R}) .
$$

Since $T_{\mu_{\alpha_{\beta}} x} \rightarrow v$, we are led to

$$
\lambda_{s}\left\langle T_{s} x, y\right\rangle=\langle v, y\rangle, \quad \forall y \in E
$$

for some invariant mean $X$. This, together with Theorem 4.1, implies that

$$
v \in \cap_{s \in S} \overline{C O}\left\{T_{t+s} x: t \in S\right\} \cap A_{C}^{g}(\mathcal{R}) .
$$

Thus we have $\left\{T_{\mu_{\alpha}}\right\}_{\alpha \in I}$ converges weakly to a point $z_{0} \in A_{C}^{g}(\mathcal{R})$. If, in addition, $C$ is closed and convex, then $z_{0} \in C$ and therefore $z_{0} \in F(\mathcal{R})$ and $\left\{T_{\mu_{\alpha}} x\right\}_{\alpha \in I}$ converges weakly to a point $z_{0} \in F(\mathcal{R})$. Following the proof of Lemma 4.3, we conclude that $z_{0}=\lim _{s} R_{F(\mathcal{R})}^{g} T_{S} x$. 


\section{REFERENCES}

[1] J.-B. Baillon, Un théorème de type ergodique pour les contractions non linéaires dans un espace de Hilbert. C. R. Acad. Sci. Paris Sér. A-B 280 (1975), 1511-1514.

[2] M.J. Borwein, Simeon Reich, Shoham Sabach, A charactrization of Bregman firmly nonexpansive operators using a new monotonicity concept, J. Nonlinear Convex Anal. 12 (2011), 161-184.

[3] L.M. Bregman, The relation method of finding the common point of convex sets and its application to the solution of problems in convex programming, USSR Comput. Math. Math. Phys. 7 (1967), 200-217.

[4] D. Butnariu, A.N. Iusem, Totally Convex Functions for Fixed Points Computation and Infinite Dimensional Optimization, Kluwer Academic Publishers, Dordrecht 2000.

[5] Y. Censor, A. Lent, An iterative row-action method for interval convex programming, J. Optim. Theory Appl. 34 (1981), 321-358.

[6] G. Chen, M. Teboulle, Convergence analysis of a proximal-like minimization algorithm using Bregman functions, SIAM J. Optim. 3 (1993), 538-543.

[7] M.M. Day, Amenable semigroups, Illinois J. Math. 1 (1957), 509-544.

[8] L. Eslamizadeh, E. Naraghirad, H.Y. Chen, Fixed point properties for semigroups of Bregman nonexpansive type mappings on unbounded sets in Banach spaces, J. Nonlinear Convex Anal. 20 (2019), 539-550.

[9] F.P. Greenleaf, Invariant Means on Topological Groups and Their Applications, Van Nostrand Mathematical Studies, vol. 16, Van Nostrand Reinhold Co., Front Cover. Frederick P. Greenleaf, 1969.

[10] N. Hirano, K. Kido, W. Takahashi, Nonexpansive retractions and nonlinear ergodic theorems in Banach spaces, Nonlinear Anal., 12 (1998), 1269-1281.

[11] P. Kocourek, W. Takahashi, J.-C. Yao, Fixed point theorems and weak convergence theorems for generalized hybrid mappings in Hilbert spaces, Taiwanese J. Math. 14 (2010), 2497-2511.

[12] F. Kohsaka, W. Takahashi, Proximal point algorithms with Bregman functions in Banach spaces, J. Nonlinear Convex Anal. 6 (2005), 505-523.

[13] A.T.-M. Lau, Invariant means on almost periodic functions and fixed point properties, Rocky Mountain J. 3 (1973), 69-76.

[14] A.T.-M. Lau, Semigroup of nonexpansive mappings on a Hilbert space, J. Math. Anal. Appl. 105 (1985), 514-522.

[15] A.T.-M. Lau, J. Ludwig, Fourier-Stieltjes algebra of a topological group, Adv. Math. 229 (2012), $2000-2023$.

[16] A.T.-M Lau, N. Shioji, W. Takahashi, Existence of nonexpansive retractions for amenable semigroups of nonexpansive mappings and nonlinear ergidic theorems in Bananch spaces, J. Funct. Anal. 161 (1999), 514522.

[17] A.T.-M Lau, W. Takahashi, Weak convergence and nonlinear ergodic theorems for reversible semigroups of nonexpansive mappings, Pacific J. Math. 12 (1987), 277-294.

[18] A.T.-M. Lau, W. Takahashi, Invariant means and fixed point properties for nonexpansive representations of topological semigroups, Topol. Methods Nonlinear Anal. 5 (1995), 39-57.

[19] A.T.-M. Lau, Y. Zhang, Fixed point properties of non-expansive mappings on weakly compact convex sets, J. Funct. Anal. 254 (2008), 2534-554.

[20] A.T.-M. Lau, Y. Zhang, Fixed point properties for semigroups of nonlinear mappings on unbounded sets, J. Math. Anal. Appl. 433 (2016), 1204-1219.

[21] V. Martín-Márquez, S. Reich, S. Sabach, Right Bregman nonexpansive operators in Banach spaces, Nonlinear Anal. 75 (2012), 5448-5465.

[22] V. Martín-Márquez, S. Reich, S. Sabach, Existence and approximation of fixed points of right Bregman nonexpansive operators, Computational and Analytical Mathematics, Springer Proc. Math. Stat.,vol. 50, pp. 501-520, Springer, New York, 2013

[23] V. Martín-Márquez, S. Reich, S. Sabach, Iterative methods for approximating fixed points of Bregman nonexpansive operators, Discrete Contin. Dyn. Syst. 6 (2013), 1043-1063.

[24] V. Martín-Márquez, S. Reich, S. Sabach, Bregman strongly nonexpansive operators in reflexive Banach spaces, J. Math. Anal. Appl., 440 (2013), 597-614.

[25] E. Naraghirad, Halpern's iteration for Bregman relatively nonexpansive mappings in Banach spaces, Numer. Funct. Anal. Optim. 34 (2013), 1129-1155. 
[26] E. Naraghirad, W. Takahashi, J.-C. Yao, Generalized retraction and fixed point theorems using Bregman functions in Banach spaces, J. Nonlinear Convex Anal. 13 (2012), 141-156.

[27] E. Naraghirad, J.C. Yao, Bregman weak relatively nonexpansive mappings in banach spaces, Fixed Point Theory Appl. 2013 (2013), 141. 1-43.

[28] A.L.T. Paterson, Amenability, American Mathematical Society, Providence, 1988.

[29] J.P. Pier, Amenable Locally Compact Groups, John Wiley and Sons, New York, 1984.

[30] W.O. Ray, The fixed point property and unbounded sets in Hilbert space, Trans. Amer. Math. Soc. 258 (1980), 531-537.

[31] S. Reich, Strong convergence theorems for resolvents of accretive operators in Banach spaces, J. Math. Anal. Appl. 75 (1980), 287-292.

[32] S. Reich, Approximating fixed points of nonexpansive mappings, Panamer Math. J. 4 (1994), 23-28.

[33] S. Reich, A limit theorem for projections, Linear and Multilinear Algebra, 13 (1983), 281-290.

[34] S. Reich, S. Sabach, Existence and approximation of fixed points of Bregman firmly nonexpansive mappings in reflexive Banach spaces, Fixed-Point Algorithms for Inverse Problems in Science and Engineering, pp. 299-314, Springer, New York, 2010.

[35] R.T. Rockafellar, On the maximal monotonicity of subdifferential mappings, Pacific J. Math. 33 (1970), 209-216.

[36] R.T. Rockafellar, On the maximality of sums of nonlinear monotone operators, Trans. Amer. Math. Soc. 149 (1970), 75-88.

[37] W. Takahashi, Nonlinear Functional Analysis, Fixed Point Theory and Its Applications, Yokahama Publishers, Yokahama, 2000.

[38] W. Takahashi, N.C. Wong, J.C. Yao, Attractive point and mean convergence theorems for semigroups of mappings without continuity in Banach spaces, J. Fixed Point Theory Appl. 16 (2014), 203-227.

[39] W. Takahashi, N.-C. Wong, J.-C. Yao, Attractive point and mean convergence theorems for semigroups of mappings without continuity in Hilbert spaces, J. Nonlinear Convex Anal. 15 (2014), 1067-1103.

[40] C. Zălinescu, Convex Analysis in General Vector Spaces, World Scientific Publishing Co. Inc., River Edge NJ, 2002. 